\section{Diagnosis of the}

\section{neuroacanthocytosis syndromes}

There are at least four neurodegenerative disorders of the basal ganglia in which acanthocytes may occur: chorea-acanthocytosis, McLeod syndrome, Huntington disease-like 2, and pantothenate kinase-associated neurodegeneration. Walker et al. review these disorders and note that while there is considerable overlap in terms of the movement disorder presentations, other clinical and laboratory features generally facilitate making the correct diagnosis.

see page 92

\section{Internal pallidal stimulation in neuroacanthocytosis}

Guehl et al. studied the effect of chronic stimulation of the internal pallidum in two patients with neuroacanthocytosis. Maximum clinical benefit was obtained with $40 \mathrm{~Hz}$ stimulation, which improved chorea without effect on hypotonia. A higher frequency $(130 \mathrm{~Hz})$ was effective on dystonia, but worsened chorea.

see page 160

\section{The incidence of anticoagulant-} associated ICH

Flaherty et al. found that the incidence of anticoagulant-associated intracerebral hemorrhage quintupled in their population during the 1990s. Among persons aged $\geq 80$ point estimates increased more than tenfold. Warfarin distribution in the United States quadrupled during the same period. Anticoagulantassociated intracerebral hemorrhage now occurs at a frequency comparable to subarachnoid hemorrhage.

see page 116

\section{Diffusion-weighted MRI in} temporal lobe epilepsy

In this study of 22 patients with temporal lobe epilepsy, Wehner et al. found that hippocampal diffusivity correlated with hippocampal atrophy. However, diffusivity measurement did not yield additional lateralizing information in the presurgical evaluation of candidates for temporal lobectomy.

see page 122

\section{Somatic symptoms and depression in women with headache}

A multicenter cross-sectional study by Tietjen et al. found a high prevalence of severe somatic symptoms in women with chronic, disabling migraine seeking specialty clinic care.

\section{Therapy for children with infantile- onset Pompe disease}

Kishnani et al. found that treatment with recombinant human acid $\alpha$-glucosidase reduced the risk of death or invasive ventilation by $92 \%$ in children $\leq 6$ months old with infantile-onset Pompe disease, thus dramatically changing the natural history of this otherwise lethal disease. The young age when they began treatment may have contributed to their good response.

see page 99

\section{Broad-spectrum of Pompe disease in patients with the same c.-32-13T $\rightarrow$ G haplotype}

Kroos et al. studied 98 cases of Pompe disease. They describe clinical heterogeneity among patients with the same c.-32-13T $\rightarrow$ G haplotype and conclude that secondary factors may substantially influence the clinical course of patients with this mutation.

see page 110

The editorial by Kathryn R. Wagner summarizes four additional diseases that may respond to enzyme replacement therapy and notes that there are few therapeutic options in neurology that produce such dramatic results as rhGAA yields in infantile Pompe disease. The findings by Kishnani et al. also raise ethical questions. These questions include whether the treatment will be converting a lethal disorder to a chronic severe disorder, what the quality of life will be for the individual and family, how end of life decisions will now be made, and possibly, whether adequate resources exist in our society for this intervention. In addition, the wide variation in the extent of ERT benefit needs to be explained. The article by Kroos et al. points to other factors influencing disease progression predicted from gene lesion or even family history.

see page 88

\section{Complex regional pain syndrome distorts visuospatial perception}

Sumitani et al. found that visual subjective bodymidline judgment of patients with complex regional pain syndrome deviates toward the affected side; however, this visuospatial deviation is reversed toward the unaffected side by therapeutic deafferentation. Complex regional pain syndrome can impair higher brain function. 


\section{Incidence of ALS in} Lombardy, Italy

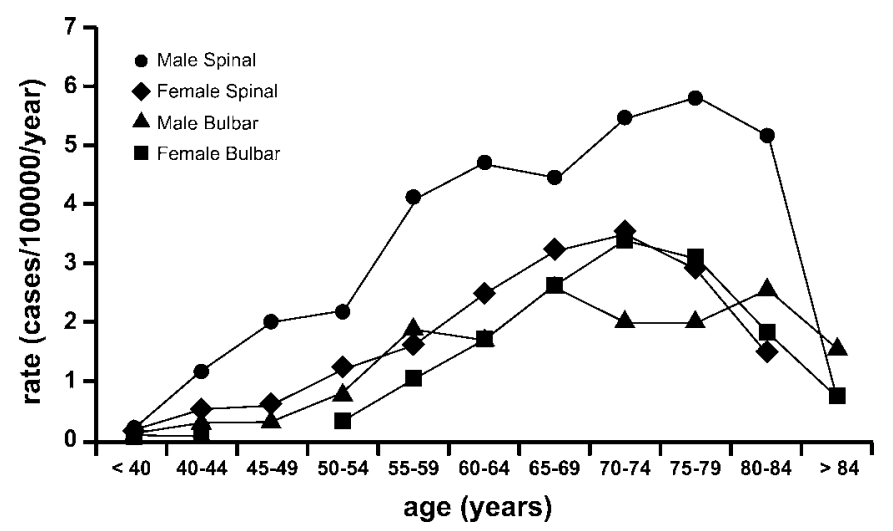

Beghi et al. enrolled 517 newly diagnosed patients with ALS during the period 1998-2002 through a prospective regional registry in Lombardy, Northern Italy. The standardized incidence rate was 2.09 per 100,000/year and varied with age, sex, and site of onset but not with time or place.

see page 141

\section{Phenylpropanolamine and hemorrhagic stroke}

The acute brain bleeding analysis (ABBA) study was a prospective case-control study to elucidate effects of small dose phenylpropanolamine (contained in cold remedies) on incident hemorrhage stroke. The data from Yoon et al. suggest that phenylpropanolamine in cold remedies increased the risk of hemorrhagic stroke, particularly in women.

\section{Limb-kinetic apraxia in PD}

Medicated patients with Parkinson disease and controls performed rapid finger tapping, measuring bradykinesiarigidity and coin-rotation, measuring precise, independent, but coordinated finger movements. Quencer et al. found that finger tapping was normal but coin rotation was impaired in PD, and suggested the term limb-kinetic apraxia for this non-dopamine responsive source of PD disability.

see page 150

The editorial by Landau and Mink notes that bradykinesia is a cardinal sign of PD. Although taken to mean "slow movements," it is apparent from clinical experience and quantitative studies that the movement impairment in $P D$ is more than simple slowing. Rhythmically repeated movements are progressively damped so that the last movement in a series is often smaller and slower than the first. Movement sequences are more impaired than individual movements. The Quencer et al. study design was simple and elegant. The results were clear; coin rotation was impaired when finger tapping was not. However, they are concerned with the mode of rationalization of mechanism for this instrumented negative symptom disability. The explanatory term "apraxia" may be easier to apply than validate. They review authoritative definitions of apraxia. While it is apparent that the motor deficit of $P D$ is more than simple slowness and stiffness, they question whether the term apraxia of impaired fine motor control provides insight into mechanism.

see page 90

\section{Intracranial pressure is measured by lumbar puncture}

Lenfeldt et al. investigated the measurability of intracranial pressure (ICP) via lumbar puncture in patients with communicating hydrocephalus. Lumbar CSF pressure and brain tissue ICP agreed well with a difference of $10 \mathrm{~mm} \mathrm{H}_{2} \mathrm{O}(0.75 \mathrm{~mm} \mathrm{Hg})$ and a regression coefficient of almost unity, demonstrating that lumbar puncture can be used to assess ICP in patients with communicating CSF systems.

see page 155

\section{ALERT: NEUROLOGY NOW USING ONLINE PEER REVIEW AND MANUSCRIPT SUBMISSION SYSTEM}

Neurology is now using an online peer review and manuscript submission system called Bench $>$ Press.

Authors should upload all original submissions via the Neurology website (www.submit.neurology.org). The Instructions to Authors detail the submission process and adjusted specifications. 


\title{
Neurology
}

\author{
January 9 Highlights \\ Neurology 2007;68;86-87 \\ DOI 10.1212/01.wnl.0000253935.81341.68
}

This information is current as of January 8, 2007

\section{Updated Information \&}

Services

Permissions \& Licensing

Reprints including high resolution figures, can be found at:

http://n.neurology.org/content/68/2/86.full

Information about reproducing this article in parts (figures,tables) or in its entirety can be found online at:

http://www.neurology.org/about/about_the_journal\#permissions

Information about ordering reprints can be found online:

http://n.neurology.org/subscribers/advertise

Neurology ${ }^{\circledR}$ is the official journal of the American Academy of Neurology. Published continuously since 1951, it is now a weekly with 48 issues per year. Copyright. All rights reserved. Print ISSN: 0028-3878. Online ISSN: 1526-632X.

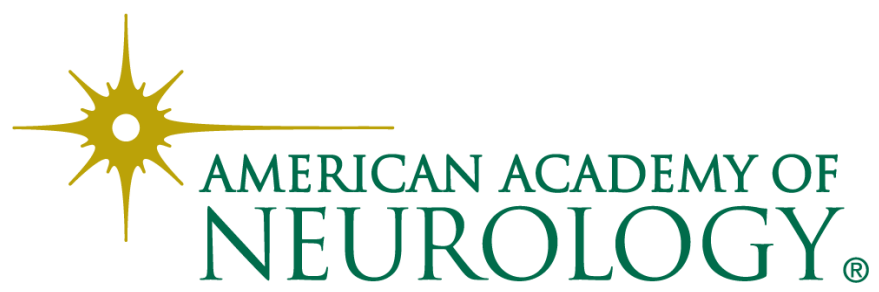

